\title{
Integrated Small RNA Sequencing, Transcriptome, and GWAS Data Reveal miRNA Regulation in Response to Milk Protein Traits in Chinese Holstein Cattle
}

\section{Wentao Cai}

Chinese Academy of Agricultural Sciences https://orcid.org/0000-0002-5639-1755

\section{Cong Li}

China Agricultural University

Junya Li

Chinese Academy of Agricultural Sciences

Jiuzhou Song

University of Maryland at College Park

Shengli Zhang ( $\sim$ zhangsicau@cau.edu.cn )

China Agricultural University

\section{Research}

Keywords: MicroRNA, Transcriptome, GWAS, Milk protein, Holstein, Milk production traits

Posted Date: January 13th, 2021

DOI: https://doi.org/10.21203/rs.3.rs-142254/v1

License: (c) (i) This work is licensed under a Creative Commons Attribution 4.0 International License.

Read Full License 
1 Integrated small RNA sequencing, transcriptome, and GWAS data

2 reveal miRNA regulation in response to milk protein traits in Chinese

3 Holstein cattle

$4 \quad$ Wentao Cai ${ }^{1,2,3}$, Cong $\mathrm{Li}^{2}$, Junya $\mathrm{Li}^{1}$, Jiuzhou Song ${ }^{3 *}$, Shengli Zhang ${ }^{2 *}$

$5 \quad{ }^{1}$ Laboratory of Molecular Biology and Bovine Breeding, Institute of Animal Science,

6 Chinese Academy of Agricultural Sciences, Beijing 100193, China

$72^{2}$ Key Laboratory of Animal Genetics, Breeding and Reproduction, Ministry of 8 Agriculture \& National Engineering Laboratory for Animal Breeding, College of

9 Animal Science and Technology, China Agricultural University, Beijing 100193, China

$10{ }^{3}$ Department of Animal and Avian Science, University of Maryland, College Park, MD 11 20742, USA

$13 *$ Corresponding author:

14 Shengli Zhang and Jiuzhou Song

15 Email address:

16 Wentao Cai: caiwentao@ caas.cn

17 Cong Li: congl@nwafu.edu.cn

18 Junya Li: lijunya@ caas.cn

19 Jiuzhou Song: songj88@ umd.edu

20 Shengli Zhang: zhangslcau@ @au.edu.cn 


\section{Abstract}

Background: Milk protein is one of the most important economic traits in the milk industry. Our previous study has revealed some functional genes responsible for milk protein synthesis in mammals. Yet, the miRNA-mediated gene regulatory network for the synthesis of milk protein in mammary is poorly understood. Results: 12 samples from Chinese Holstein Cows with three too high and three low phenotypic values for milk protein percentage in lactation and non-lactating were examined through deep small RNA sequencing. By bioinformatics analysis, we characterized 387 known and 212 novel miRNAs in the mammary gland. Differentially expressed analysis detected 28 miRNAs in lactation and 52 miRNAs in the non-lactating period with a highly significant correlation with milk protein concentration. Target prediction and correlation analysis identified some key miRNAs and their targets potentially involved in the synthesis of milk protein. Using genome-wide association signal (GWAS) enrichment analysis among five milk production traits, we found the differentially expressed targets were significantly related to milk protein traits.

Conclusions: This integrated study on the transcriptional and post-transcriptional regulatory profiles between significantly differential phenotype of milk protein concentration provides new insights into the mechanism of milk protein synthesis, which should reveal the regulatory mechanisms of milk secretion.

Keywords: MicroRNA, Transcriptome, GWAS, Milk protein, Holstein, Milk production traits 


\section{Introduction}

Milk protein is one of the best protein sources for humans[1]. It also affects milk manufacturing properties such as cheese yields, milk coagulation time, and curd firmness $[2,3]$. Improving milk protein yields and quality can increase the economic outcome of the dairy industry. It has been reported that the amount and compositions of proteins in milk are determined mainly by genetic factors [2]. The heritabilities of milk protein compositions were moderate to high in Dutch Holstein-Friesian cattle, ranging from 0.25 to 0.80 [4]. So far, several strategies, such as QTL mapping, candidate gene analysis, genome-wide association studies (GWAS), or next-generation sequencing (NGS) technologies [5-8], have been adopted to increase milk protein yields and compositions. However, the synthesis and secretion of milk proteins involve complex physiological and biochemical processes. One of the mechanisms is microRNAs' roles, which need to be thoroughly examined.

MicroRNAs (miRNAs) are a class of small (18-24 nucleotide) RNAs that involve in the regulation of gene expression by targeting messenger RNAs (mRNA). The vast majority of miRNA genes are transcribed by the RNA polymerase II, which generates long primary transcripts (pri-miRNA) that contain a hairpin stem-loop structure [9]. miRNAs are processed from double-stranded hairpin precursors by Drosha protein in the nucleus and Dicer protein in the cytoplasm. The final single-stranded mature miRNA hybridizes with the RNA-induced silencing complex (RISC) to undergo gene inhibition $[10,11]$. Unlike other regulators, miRNAs exert highly complex combinatorial gene regulations by targeting hundreds of mRNA transcripts[12]. Extensive research in the past decade indicates miRNAs' involvement in various biological processes such as cell development, proliferation, differentiation, and apoptosis[13-15]. Recently, miRNAs have been shown to play important roles in the milk secretion process through their altered regulation of genes involved in milk protein and fat synthesis[16, 17]. Fifty-six mammary miRNAs in the lactation showed significant differences in expression compared to non-lactating in Holstein cattle [18]. Several miRNAs, such as miR-15a [19], miR-139 [20], miR-423-5p [21], miR-101b [22], miR-486 [23], miR-152 [24], miR-135 [25] and miR-138 [18] appear to affect 
milk protein synthesis by regulating key genes of protein synthesis pathways. Although the identification and characterization of miRNA in mammals of bovine have been reported [18, 26-28], to our knowledge, only a few studies describe miRNA profiles specific to the synthesis of milk protein in bovine. The inspiration of many miRNAs studies in milk protein synthesis in bovine was from other species [19], some even from another biological process that was unknown in mammary tissue before [20, 23]. The real miRNA profiles specific to milk protein traits are limited in bovine.

In this study, the hypothesis is that miRNAs have potential roles in mammary milk protein production. Using miRNA-seq and RNA-seq, we investigated 12 bovine mammary glands from Holstein cows with too high or low milk protein percentage at peak lactation and during the non-lactating period, respectively. We believe that the results from the integrated transcriptome analyses of miRNA, mRNA, and previous GWAS information will help us identify new miRNA related to milk protein, further enhancing our understanding of the milk synthesis mechanisms.

\section{Methods}

\section{Mammary samples}

Based on Dairy Herd Improvement system (DHI) data, the 12 multiparous and healthy mastitis-free Chinese Holstein cattle with three too high and three low phenotypic values for milk protein percentage peak and non-lactation period were chosen from our study, which has been described in the previous study[29]. All mammary samples were retrieved from the 12 candidate Chinese Holstein cows using a biopsy, then placed in liquid nitrogen and subsequently stored at $-80{ }^{\circ} \mathrm{C}$ until RNA isolation.

\section{RNA extraction and library preparation for Small RNA sequencing}

Total RNA was extracted using TRIzol reagent (Invitrogen, Carlsbad, CA, USA). Twelve small RNA libraries from RNA integrity and concentration were assessed using the RNA Nano 6000 Assay Kit of the Bioanalyzer 2100 system (Agilent Technologies, CA, USA). All RNA samples had an RNA integrity number of at least 7.5. 15\% agarose gels separated total RNA to extract the small RNA (18-30 nt). After precipitated by 
ethanol and centrifugal enrichment of small RNA sample, the library was prepared according to the method and process of Small RNA Sample Preparation Kit (Illumina, RS-200-0048). RNA concentration of library was measured using Qubit® RNA Assay Kit in Qubit® 2.0 to preliminary quantify and then dilute to $1 \mathrm{ng} / \mu \mathrm{l}$. Insert size was assessed using the Agilent Bioanalyzer 2100 system (Agilent Technologies, CA, USA). After the insert size consistent with expectations, the qualified insert size was accurate quantitative using the Taqman fluorescence probe of AB Step One Plus Real-Time PCR system (Library valid concentration $>2 \mathrm{nM}$ ). The qualified libraries were sequenced by an Illumina Hiseq 2500 platform and generate $50 \mathrm{bp}$ single-end reads.

\section{Bioinformatics analysis of small RNAs}

Quality trimming and adaptor removal of the Illumina reads were carried out using Cutadapt and Trimmomatic[30, 31]. After filtering for their size (18-30 nt), the cleaned reads were categorized into unique tags and then mapped to the bovine (UMD3.1.1) reference genomes by the bowtie allowed one mismatch to analyze their expression and distribution on the reference[32]. All the downstream analyses were based on the mapped small RNA tags.

The matching sequences ranged from $18 \mathrm{nt}$ to $30 \mathrm{nt}$ were used to align against the miRBase 21.0 (http://www.mirbase.org/) to identify known miRNAs by miRDeep2 with a quantifier.pl module[33]. The sequences matching other small RNAs, including rRNA, snRNA, repeat RNA, tRNA and snoRNA, were compared with Bos taurus noncoding RNA sequences in the Sanger RNA family database (Rfam 12.1) using infernal $1.1[34,35]$. Unannotated sequences combined with the known miRNA annotation from Ovis aries, Capra hircus, Sus scrofa, Mus musculus, and Homo sapiens were used to predict the novel miRNAs according to the characteristic hairpin structure of microRNA precursors by miRDeep2 core module miRDeep2.pl. We characterized miRNAs expressed at least two samples as novel miRNAs. To make every unique small RNA mapped to only one annotation, we followed the priority rule: known miRNA > rRNA $>$ tRNA $>$ snRNA $>$ snoRNA $>$ repeat $>$ novel miRNA $>$ ta-siRNA.

\section{Differential expression analysis}

To investigate differentially expressed (DE) miRNAs between high and low milk 
protein percentage during peak and non-lactating periods (i.e., HP vs. LP, HD vs. LD) using the DESeq2 $\mathrm{R}$ package[36]. MiRNAs with a p-value $<0.05$ and $\mid \log _{2}$ (foldchange) $\mid>0.8$ were assigned as differentially expressed. The expression patterns of DE miRNAs across four groups were performed using the k-mean method [37]. Using gap statistics, we determined that $\mathrm{k}=7$ was the optimal choice for distinguishing these miRNAs.

\section{MiRNA function prediction and regulatory network construction}

We predicted the binding of differentially expressed miRNAs to the putative targets using miRanda with default parameters[38]. The predicted target genes were compared with the previous transcriptome profiling data, and only genes that are inversely correlated in expression with the targeting miRNA were included[29]. Analysis to identify negative correlations between miRNA and mRNA expression was done using an in-house R script. Briefly, normalized expression of miRNA and mRNA expression were sample-matched for all samples. Then for each miRNA, Pearson correlation coefficients were computed for all mRNAs with p-value $<0.05$. To evaluate the miRNA-gene regulatory network, target genes were annotated using DAVID (https://david.ncifcrf.gov/) for assigning GO terms and KEGG pathways to investigate putative functions[39]. The statistical significance of GO term or KEGG pathway enrichment was measured by Fisher's exact test with p-value $<0.05$. After the correlation mapping, miRNAs and their targeting genes were subjected to the network visualization analysis. The Cytoscape software was used to construct the network[40].

\section{The enrichment analysis of GWAS signals}

We obtained summary statistics of single-trait GWAS for five milk production traits, heifer conception rate, and somatic cell score in cattle described previously[41]. Here we provide a summary. The de-regressed PTAs (predicted transmitting abilities) were used as a phenotype in all seven traits. SNPs and insertion-deletion (InDel) calls were from Run 5 of the 1000 Bull Genomes Project. After sequence markers impute and quality control, genotypes of 3,148,506 sequence variants for 27,214 Holstein bulls were obtained. The single-trait GWAS analyses were conducted using a mixed-model approach by MMAP (https://mmap.github.io/). 
We applied a sum-based method for GWAS signals enrichment analyses across targets of DE miRNAs. The sum-based method uses signals of all markers within a pre-defined list of target genes. Briefly, we calculated the following summary statistics for the targets group of DE miRNAs:

$$
T_{\text {sum }}=\sum_{i=1}^{m_{g}} \beta^{2}
$$

In which, $T_{\text {sum }}$ is the summary statistics for a tested gene group. $m_{g}$ is the number of SNPs located in target genes or $5 \mathrm{~kb}$ upstream/downstream of target genes, and $\beta$ is the estimate of marker effect obtained in GWAS statistics. The SNP markers located in genes (including $5 \mathrm{~kb}$ up and downstream) were numbered using $1 \ldots N$. The observed SNPs located in all targets were $N_{1}, N_{2}, N_{3}, \ldots, N_{n}$. Their test statistics were $\beta_{N_{1}}{ }^{2}$, $\beta_{N_{2}}{ }^{2}, \beta_{N_{3}}{ }^{2}, \ldots, \beta_{N_{n}}{ }^{2}$. For the permutation test, we chose number $\mathrm{R}$ within $1 \sim \mathrm{N}$. Then the observed SNPs set was shifted to the new rank order $\left(P_{1}, P_{2}, P_{3}, \ldots, P_{n}\right)$ based on random number $\mathrm{R}$ using the following formula:

$$
P_{i}=\left\{\begin{array}{cc}
N_{i}+R, & N_{i}+R \leq \mathrm{N} \\
N_{i}+R-\mathrm{N}, & N_{i}+R>\mathrm{N}
\end{array}\right.
$$

All test statistics were moved to the new positions, with the remaining markers maintaining the original order. A new summary statistic of a genomic feature $\left(\beta_{P_{1}}{ }^{2}, \beta_{P_{2}}{ }^{2},{\beta_{P_{3}}}^{2}, \ldots, \beta_{P_{n}}{ }^{2}\right)$ was calculated based on the original position of the feature. The permutation was repeated 1,0000 times for each studied genomic feature, and an empirical P-value was then calculated based on one-tailed tests of the proportion of randomly sampled summary statistics larger than that observed using the following formula:

$$
\mathrm{P}=\left(N_{\text {over }}+1\right) / 10001
$$

Where $N_{\text {over }}$ represents the times of the permutated $T_{\text {sum }}$ large than the genome feature $T_{\text {sum }}$. To avoid the bias by DGAT1 genes, the SNPs located in DGAT1 gene or $1 \mathrm{Mb}$ upstream/downstream extended region were removed. This sum-based method for GWAS signals enrichment analyses using Perl scripts have been available (https://github.com/WentaoCai/GWAS_enrichment). 


\section{Results}

\section{Overview over small RNA sequencing}

195

To study miRNAs in milk protein synthesis's complex process, we profiled miRNA changes between the high milk protein percentage and low milk protein percentage groups in both lactation and non-lactating period using small RNA sequencing. After trimming adaptor sequences and removing contaminated reads, an average of 23.0 million clean reads ranges from 22.4 to 23.9 million were generated. Then we categorized them into unique tags; an average of 1.1 million unique tags was obtained (Table S1). We separately mapped clean reads and unique tags to the bovine (UMD3.1.1) reference genomes. The mapping rate was about $90.0 \%$ and $74.4 \%$ using total clean reads and unique tags, respectively (Table S2). The majority of the mapped reads ranged from 21 to $23 \mathrm{nt}$ in length, and the $22 \mathrm{nt}$ small RNA was the most abundant (Figure 1a). As expected, most reads were observed to match with 3'-UTR and 5'-UTR region allocating microRNAs (Figure $1 \mathrm{~b}$ ). These results confirm the reliability of the small RNA sequencing process used in our study. The residual fraction of mapped reads not corresponding to microRNAs was distributed among a miscellanea of annotated regions, including rRNAs (14.76\%), tRNAs (3.48\%), snRNAs (0.38\%), snRNAs $(0.78 \%)$, and repeats $(0.04 \%)$ (Figure $1 \mathrm{c})$.

\section{Identification of known and novel miRNAs}

We identified 387 known expressed miRNAs in the 12 mammary samples (TPM > 0.5). The clustering heatmap of miRNA expression profiles derived from the samples is shown in Figure 2a. We also compared the miRNAs with the greatest expression (top 20) in the mammary tissue at lactation and non-lactating periods (Figure S1). The top expressed miRNAs in both of the two stages were similar except for miR-142 and miR126, which were explicitly expressed higher in the lactation stage and non-lactating period, respectively. The most significant expression of miRNAs in lactation was miR148a, while miR-143 was the most highly expressed in the non-lactating period. We characterized 415 novel miRNAs, including 235 and 254 novel miRNAs identified in 
the lactation and non-lactating periods. Interestingly, we found 16 novel miRNAs expressed in all 12 samples (Table S3).

Differentially expressed miRNA within extremely phenotype in lactation and nonlactating period

In the twelve miRNA libraries, 28 differentially expressed (DE) miRNAs were identified between HP and LP groups in lactation, including 11 upregulated and 17 downregulated miRNAs in the LP group relative to the HP group (p-value $<0.05$, $\log 2$ foldchange $>0.8$, as shown in Figure $2 \mathrm{~b}$ and $2 \mathrm{c}$ ). A total of 52 miRNAs were differentially expressed between HD and LD groups in non-lactating period, including 22 up-regulated and 30 down-regulated miRNAs in LD group relative to HD group. Interestingly, we found 14 differentially expressed miRNAs exhibited common changes across the two comparison groups (Table 1).

Trends in DE miRNAs in lactation or non-lactating were examined using k-means clustering, which revealed that 66 DE miRNAs could be divided into seven distinct clusters with differentially expression level pattern changes (Figure 3). Cluster 1, cluster 5, and cluster 6 9 revealed that the expression change pattern of miRNAs in HP vs. LP was similar to HD vs. LD, including 14 common miRNAs significantly changing in expression levels in lactation remained altered in the same direction in the nonlactating period.

\section{Target gene prediction of differentially expressed miRNAs}

To better understand the DE miRNAs' functions, putative target genes were predicted using the 3'-UTR sequence of mRNA by the miRanda software. We predicted 9,156 target mRNAs for the 28 DE miRNAs in HP vs. LP and 10,045 target mRNAs for the 52 DE miRNAs in HD vs. LD (Table S4). To identify target genes with high confidence, we performed a correlation analysis between expression levels of the DE miRNAs and the expression levels of target genes in 12 mammary samples. The expression of target genes from the 12 same samples was quantified by mRNA sequencing mentioned in our previous study[29]. We detected 28 DE miRNAs inversely correlated with 1685 targets resulted in 2468 miRNA-mRNA pairs for HP vs. LP, and 52 DE miRNAs inversely correlated with 2280 targets resulted in 3697 miRNA- 
mRNA pairs for HD vs. LD. For the 14 common DE miRNAs across HP vs. LP and HD vs. LD, we found 914 inversely correlated target mRNAs of common DE miRNAs between HP vs. LP and HD vs. LD resulted in 1210 miRNA-mRNA pairs (p-value $<0.05)$.

\section{Functional annotation of differentially expressed miRNAs}

To functionally classify the DE miRNAs, GO and KEGG enrichment analysis were performed for DE miRNAs' confident target genes in peak lactation and nonlactating period, respectively. Pathway analysis showed that these 914 target genes of common DE miRNAs were significantly enriched in 60 pathways. Many pathways were associated with protein syntheses, such as insulin secretion, mTOR signaling pathway, estrogen signaling pathway, insulin signaling pathway, and GnRH signaling pathway. Gene Ontology analysis demonstrated these target genes were involved in protein transport, trans-Golgi network, metabolic process, protein serine/threonine kinase activity (Table S5).

Their target genes were enriched in mTOR signaling pathway, TNF signaling pathway, Leukocyte transendothelial migration, and MAPK signaling pathway for specifically DE miRNAs in peak lactation. Significantly functional terms were noticed for positive regulation of transcription, post-Golgi vesicle-mediated transport, mRNA 3'-UTR binding, ER to Golgi transport vesicle. For DE miRNAs in the non-lactating period, several target genes were observed to be involved in the PI3K-Akt signaling pathway, Metabolic pathways, and mTOR signaling pathway. Their functions were associated with protein transport, transcription, vasculogenesis, positive regulation of gene silencing by miRNA. The KEGG pathways and top 10 terms for GO categories were shown in Figure $4 a$.

\section{Regulatory networks for differentially miRNAs-mRNAs}

To better understand the relationship between miRNAs and milk protein traits, we selectively analyzed the 214 miRNA-mRNA pairs. Both miRNAs and their targets were differentially expressed in HP vs. LP or HD vs. LD. We found 22 DE miRNAs potentially regulated 24 DEGs were involved in milk protein synthesis (Table 2). For example, $P S P H$, a significantly dysregulated milk protein gene, was involved in the 
biosynthesis of amino acids, metabolic pathways, and glycine, serine \& threonine metabolism. The expression of miR-1 was negatively correlated with PSPH. FABP3, targeted by miR-146b and miR-185, influences fat and protein content in cattle. Additional genes are listed in Table 2. Besides, we believed that we were able to account for all known target genes involved in milk protein synthesis through various pathways (Figure 4b).

\section{Validated by GWAS signals}

To assess whether DE miRNAs were associated with GWAS signals, we applied enrichment analysis from GWAS for all correlated targets of DE miRNAs across five milk traits, one reproduction trait (heifer conception rate), one health trait (somatic cell sore, SCS). All targets of DE miRNAs were considered a genome feature. Only SNPs located in targets or $5 \mathrm{~Kb}$ extended region of targets were included in calculating the squares of their effects. For comparison, 10,000 random SNPs sets located in all genes or $5 \mathrm{~Kb}$ extended areas of the bovine genome were generated. As shown in Figure 5a, the correlated targets of DE miRNAs were enriched with GWAS signals of milk protein $(P<0.1)$ and milk protein percentages $(P<0.01)$ traits. After removing all SNPs close to the well-known milk/fat gene, DGAT1, on Bos taurus chromosome 14, significant $(P<0.05)$ enrichments were observed for all five milk production traits in HP vs. LP. For the targets of DE miRNAs in HD vs. LD, significant enrichments were kept for milk protein and SCS traits. More association signals of five milk production traits were enriched in targets of upregulated miRNAs in lactation. In comparison, more association signals of milk production traits were increased in targets of down-regulated miRNAs for the non-lactating period (Figure 5b).

\section{Discussion}

Deciphering miRNA targets is crucial for molecular breeding since miRNAs are involved in critical biological processes and affect many traits. The miRNA-mediated gene regulatory network might remarkably contribute to the synthesis of milk protein. However, genome-wide identification of miRNAs specific to milk protein still lacks in the mammary of bovine. In this study, high-throughput sequencing technologies were 
used to identify candidate miRNAs and networks related to milk protein. The mammary transcriptome profiles of miRNAs in Holstein cattle with extreme phenotypes in milk protein percentage were presented.

We identified 387 miRNAs in the mammary gland, accounting for $48.6 \%$ of all known bovine miRNAs deposited in miRbase 21. A total of 485 novel miRNAs were detected in this study, which will considerably increase bovine miRNAs' repertoire. The differentially regulated expression patterns of miRNAs in mammary gland tissue underscores that the synthesis and secretion of milk protein involves a high level of post-transcriptional regulation of gene expression by miRNAs. The 14 differentially expressed miRNAs between high and low milk protein percentages across both lactation and non-lactating periods suggest that these miRNAs may partially regulate the functions of the same biological or physiological processes in the two periods.

We combined predicted miRNA target genes with the previously obtained gene expressions. We matched 1685 inversely correlated target genes that resulted in 2468 miRNA-mRNA pairs for HP vs. LP, and 2280 inversely correlated target genes resulted in 3697 miRNA-mRNA pairs for HD vs. LD. Functional annotation showed that these target genes of common DE miRNAs across two stages were associated with protein synthesis, such as mTOR signaling pathway, estrogen signaling pathway, insulin signaling pathway and GnRH signaling pathway, implying that these miRNAs could be critical players in these pathways. It should be noted that some of the common DE miRNAs in this study have been previously suggested to play essential roles in milk protein synthesis. For example, miR-152 negatively regulates DNA methyltransferase 1 (DNMT1), decreasing the global DNA methylation and increasing the expression of serine/threonine protein kinase Akt $(A K T)$ and peroxisome proliferator-activated receptor gamma $(P P A R \gamma)[24]$. These target genes of DE miRNAs, specifically for lactating, were involved in positive transcription, mRNA 3'-UTR binding, and ER to Golgi transport vesicle. Also, miR-423-5p has been shown to regulate AMPKgamma1 (AMPK $\gamma 1$ ) negatively. The 3'-UTR SNP of $A M P K \gamma 1$ was influential on the milk and protein yield traits. This mutation also deviated target mRNA base-pairing to the miR-423-5p, which implied miR-423-5p plays an important role in milk metabolism 
pathways[42]. These target genes of DE miRNAs, especially for the non-lactating period, were also associated with some milk protein metabolisms, such as PI3K-Akt signaling pathway, metabolic pathways, and mTOR signaling pathway. For example, miR-486 directly downregulates PTEN gene expression, altering the expression of downstream genes, such as $A K T$ and $m T O R$. miR-486 as a downstream regulator of PTEN required for the development of the cow mammary gland[23].

The DE miRNAs-DEGs regulatory networks provided a comprehensive profile for understanding the mechanism of milk protein synthesis in cows. Twenty-two DE miRNAs potentially regulated 24 DEGs associated with milk protein metabolism were identified. MiR-1 is a known suppressor involved in PI3K-AKT, mTOR and NFאB Pathways[43]. miR-1 controls cholesterol synthesis and regulates mammary proliferation by targeting $I G F 1$ and $T B X 3$ in the sow's mammary gland [44]. Here, we found the expression of miR-1 was negatively correlated with $P S P H$, which is an insulin-responsive gene in bovine mammary that are involved in protein synthesis[45]. Besides, proteins encoded by phosphoserine phosphatase (PSPH) are engaged in serine synthesis[46, 47]. miR-146b was upregulated in the mammary glands of the HP group, which was reported to be involved mainly in leukemia, epidermal growth factor receptor (EGFR) signaling, MAPK, and nuclear factor kappa-light-chain-enhancer of

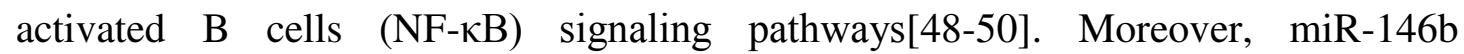
was associated with mammary gland development and stem cell activity[51]. The expression of $F A B P 3$ was negatively correlated with miR-146b. SNPs within FABP3 have been reported to influence fat and protein content in cattle [52]. These findings indicated that the expression change in DEGs and DE miRNAs within networks might contribute to milk protein metabolism in cows.

We integrated miRNA-targets with GWAS data using the sum-based marker-set test method, which has been demonstrated to have higher power or at least equal to most commonly used marker-set test methods in polygenic traits[53, 54]. Our analysis revealed significant enrichment of GWAS signals in proximity to DE miRNAs' target genes, especially to DE miRNAs in lactation, which implied the miRNAs of lactation were more associated with milk production traits. Negative genetic correlations were 
371 found between milk production traits and fertility traits, which can explain that the

372 GWAS signals of heifer conception rate trait were not enriched in targets of milk protein

373 associated miRNAs $[55,56]$. Previous studies have reported that the DEGs in non-

374 lactating periods could help the mammary tissue prevent issues with inflammation and 375 udder disorders[29]. Of interest, we found the DE miRNAs of the non-lactating period 376 were related to the SCS trait. The differences in enrichments of up/down-regulated 377 miRNAs between lactation and non-lactating period indicated the miRNAs might have 378 different patterns of regulation involved in milk-related activities.

\section{Conclusions}

This study integrated small RNA sequencing with transcriptome of mammary to detect genes/pathways associated with milk protein synthesis in cows. We provide genomic evidence that target genes of DE miRNAs were more associated with milk production traits in lactating mammary than non-lactating mammary using GWAS data. Responsive miRNAs in the mammary gland played roles in the regulation of the milk concentration, allowing for more effective breeding strategies.

\section{List of abbreviations}

DE: Differentially expressed

DEG: Differentially expressed genes

DGAT1: Diacylglycerol O-Acyltransferase 1

DHI: Dairy Herd Improvement system

EGFR: epidermal growth factor receptor

FABP3: Fatty Acid Binding Protein 3

GO: Gene ontology

400 GWAS: Genome-wide association studies 
402 HD: High milk protein percentage in dry (non-lactating) period

403 HP: High milk protein percentage in peak period (lactation)

404 IGF1: Insulin-like growth factor 1

405 KEGG: Kyoto encyclopedia of genes and genomes

406 LD: Low milk protein percentage in dry (non-lactating) period

407 LP: Low milk protein percentage in peak period (lactation)

408 MAPK: A mitogen-activated protein kinase

409 mTOR: Mammalian target of rapamycin

410 NGS: Next generation sequencing

411 PSPH: Phosphoserine phosphatase

412 PTAs: Predicted transmitting abilities

413 PTEN: Phosphatase and tensin homolog

414 QTL: Quantitative trait locus

415 RISC: RNA-induced silencing complex

416 SCS: Somatic cell sore

417 SNP: Single nucleotide polymorphism

418 TBX3: T-Box transcription factor 3

419 TNF: Tumor necrosis factor

421 Declarations

422 Ethics approval and consent to participate

423 All animal experiments were performed following the recommendations in the 424 Guide for the Care and Use of Laboratory Animals of China. The study protocol was 425 approved by the College of Animal Science and Technology, China Agricultural 426 University (Permit Number: DK996).

427 Consent for publication

428 Not applicable

429 Availability of data and materials 
The sequencing data have been deposited in NCBI/SRA database under accession number of PRJNA689373. Other data used and/or analyzed during the current study are available from the corresponding authors on reasonable request.

\section{Competing interests}

The authors declare that they have no competing interests.

\section{Funding}

This work is supported by the 863 projects (2013AA102504), National Key Technologies R \& D Program (2012BAD12B01), Beijing Dairy Industry Innovation Team, China Agricultural Research System (CARS-37), and Chinese Academy of Agricultural Sciences Foundation (2020-YWF-YB-02). We are deeply grateful to all donors who participated in this program.

\section{Authors' contributions}

SZ, JS, and JL conceived and designed the study and revised the manuscript. WC performed the small RNA related experiments, data analysis and drafted the manuscript. All authors read and approved the final manuscript.

\section{Acknowledgments}

We appreciate the Beijing Dairy Cattle Center and Beijing Sanyuanlvhe Dairy Farming Center for providing the mammary samples of Chinese Holstein.

\section{Reference}

1. Anderson GH, Tecimer SN, Shah D, Zafar TA: Protein source, quantity, and time of consumption determine the effect of proteins on short-term food intake in young men. J Nutr 2004, 134(11):3011-3015.

2. Auldist MJ, Johnston KA, White NJ, Fitzsimons WP, Boland MJ: A comparison of the composition, coagulation characteristics and cheesemaking capacity of milk from Friesian and Jersey dairy cows. Journal of Dairy Research 2004, 71(1):51-57.

3. Wedholm A, Larsen LB, Lindmark-Månsson H, Karlsson AH, Andrén A: Effect of Protein Composition on the Cheese-Making Properties of Milk from Individual Dairy Cows. Journal of Dairy Science 2006, 89(9):3296-3305.

4. Schopen GC, Heck JM, Bovenhuis H, Visker MH, van Valenberg HJ, van Arendonk JA: Genetic parameters for major milk proteins in Dutch Holstein-Friesians. J Dairy Sci 2009, 92(3):11821191.

5. Georges M, Nielsen D, Mackinnon M, Mishra A, Okimoto R, Pasquino AT, Sargeant LS, Sorensen 
A, Steele MR, Zhao X: Mapping quantitative trait loci controlling milk production in dairy cattle by exploiting progeny testing. Genetics 1995, 139(2):907-920.

6. Gambra R, Penagaricano F, Kropp J, Khateeb K, Weigel KA, Lucey J, Khatib H: Genomic architecture of bovine kappa-casein and beta-lactoglobulin. J Dairy Sci 2013, 96(8):5333-5343.

7. Li C, Cai W, Zhou C, Yin H, Zhang Z, Loor JJ, Sun D, Zhang Q, Liu J, Zhang S: RNA-Seq reveals 10 novel promising candidate genes affecting milk protein concentration in the Chinese Holstein population. Sci Rep 2016, 6:26813.

8. Zhou C, Li C, Cai W, Liu S, Yin H, Shi S, Zhang Q, Zhang S: Genome-Wide Association Study for Milk Protein Composition Traits in a Chinese Holstein Population Using a Single-Step Approach. Front Genet 2019, 10:72.

9. Lee Y, Ahn C, Han J, Choi H, Kim J, Yim J, Lee J, Provost P, Rådmark O, Kim S: The nuclear RNase III Drosha initiates microRNA processing. Nature 2003, 425(6956):415.

10. Robb GB, Rana TM: RNA helicase A interacts with RISC in human cells and functions in RISC loading. Molecular cell 2007, 26(4):523-537.

11. Kim VN, Han J, Siomi MC: Biogenesis of small RNAs in animals. Nature reviews Molecular cell biology 2009, 10(2):126.

12. Shivdasani RA: MicroRNAs: regulators of gene expression and cell differentiation. Blood 2006, 108(12):3646-3653.

13. Zhou B, Wang S, Mayr C, Bartel DP, Lodish HF: miR-150, a microRNA expressed in mature B and $\mathrm{T}$ cells, blocks early B cell development when expressed prematurely. Proc Natl Acad Sci U S A 2007, 104(17):7080-7085.

14. Gu J, lyer VR: PI3K signaling and miRNA expression during the response of quiescent human fibroblasts to distinct proliferative stimuli. Genome biology 2006, 7(5):R42-R42.

15. Johnson SM, Grosshans H, Shingara J, Byrom M, Jarvis R, Cheng A, Labourier E, Reinert KL, Brown D, Slack FJ: RAS is regulated by the let-7 microRNA family. Cell 2005, 120(5):635-647.

16. Wang D, Liang G, Wang B, Sun H, Liu J, Guan LL: Systematic microRNAome profiling reveals the roles of microRNAs in milk protein metabolism and quality: insights on low-quality forage utilization. Sci Rep-Uk 2016, 6:21194-21194.

17. Cui X, Yao M, Zhang S, Zhang Q, WU C, Sun D: Comprehensive microRNA expression profile of the mammary gland in lactating dairy cows with extremely different milk protein and fat percentages. Frontiers in Genetics 2020, 11:1504.

18. Li Z, Liu H, Jin X, Lo L, Liu J: Expression profiles of microRNAs from lactating and non-lactating bovine mammary glands and identification of miRNA related to lactation. BMC Genomics 2012, 13(1):731.

19. Li HM, Wang CM, Li QZ, Gao XJ: MiR-15a decreases bovine mammary epithelial cell viability and lactation and regulates growth hormone receptor expression. Molecules 2012, 17(10):12037-12048.

20. Cui Y, Sun X, Jin L, Yu G, Li Q, Gao X, Ao J, Wang C: MiR-139 suppresses beta-casein synthesis and proliferation in bovine mammary epithelial cells by targeting the GHR and IGF1R signaling pathways. BMC Vet Res 2017, 13(1):350.

21. Mahmoudi A, Zargaran A, Amini HR, Assadi A, Vajdi Hokmabad R, Eghbalsaied S: A SNP in the 3'-untranslated region of AMPKgamma1 may associate with serum ketone body and milk production of Holstein dairy cows. Gene 2015, 574(1):48-52.

22. Tanaka T, Haneda S, Imakawa K, Sakai S, Nagaoka K: A microRNA, miR-101a, controls 
mammary gland development by regulating cyclooxygenase-2 expression. Differentiation 2009, 77(2):181-187.

23. Li D, Xie X, Wang J, Bian Y, Li Q, Gao X, Wang C: MiR-486 regulates lactation and targets the PTEN gene in cow mammary glands. PLoS One 2015, 10(3):e0118284.

24. Wang J, Bian Y, Wang Z, Li D, Wang C, Li Q, Gao X: MicroRNA-152 regulates DNA methyltransferase 1 and is involved in the development and lactation of mammary glands in dairy cows. PLoS One 2014, 9(7):e101358.

25. Ji Z, Dong F, Wang G, Hou L, Liu Z, Chao T, Wang J: miR-135a Targets and Regulates Prolactin Receptor Gene in Goat Mammary Epithelial Cells. DNA Cell Biol 2015, 34(8):534-540.

26. Le Guillou S, Marthey S, Laloe D, Laubier J, Mobuchon L, Leroux C, Le Provost F: Characterisation and comparison of lactating mouse and bovine mammary gland miRNomes. PLoS One 2014, 9(3):e91938.

27. Wang D, Liang G, Wang B, Sun H, Liu J, Guan le L: Systematic microRNAome profiling reveals the roles of microRNAs in milk protein metabolism and quality: insights on low-quality forage utilization. Sci Rep 2016, 6:21194.

28. Li R, Beaudoin F, Ammah AA, Bissonnette N, Benchaar C, Zhao X, Lei C, Ibeagha-Awemu EM: Deep sequencing shows microRNA involvement in bovine mammary gland adaptation to diets supplemented with linseed oil or safflower oil. BMC Genomics 2015, 16:884.

29. Li C, Cai W, Zhou C, Yin H, Zhang Z, Loor JJ, Sun D, Zhang Q, Liu J, Zhang S: RNA-Seq reveals 10 novel promising candidate genes affecting milk protein concentration in the Chinese Holstein population. Sci Rep-Uk 2016, 6(1):26813.

30. Bolger AM, Lohse M, Usadel B: Trimmomatic: a flexible trimmer for Illumina sequence data. Bioinformatics 2014, 30(15):2114-2120.

31. Martin $\mathrm{M}$ : Cutadapt removes adapter sequences from high-throughput sequencing reads. EMBnetjournal; Vol 17, No 1: Next Generation Sequencing Data AnalysisDO 1014806/ej171200 2011.

32. Langmead B: Aligning short sequencing reads with Bowtie. Current protocols in bioinformatics 2010, 32(1):11.17. 11-11.17. 14.

33. Mackowiak SD: Identification of novel and known miRNAs in deep - sequencing data with miRDeep2. Current protocols in bioinformatics 2011, 36(1):12.10. 11-12.10. 15.

34. Nawrocki EP, Eddy SR: Infernal 1.1: 100-fold faster RNA homology searches. Bioinformatics 2013, 29(22):2933-2935.

35. Griffiths-Jones S, Bateman A, Marshall M, Khanna A, Eddy SR: Rfam: an RNA family database.

36. Love MI, Huber W, Anders S: Moderated estimation of fold change and dispersion for RNAseq data with DESeq2. Genome biology 2014, 15(12):550.

37. Ahmad A, Dey L: A k-mean clustering algorithm for mixed numeric and categorical data. Data \& Knowledge Engineering 2007, 63(2):503-527.

38. Enright AJ, John B, Gaul U, Tuschl T, Sander C, Marks DS: MicroRNA targets in Drosophila. Genome Biology 2003, 5(1):R1.

39. Huang DW, Sherman BT, Tan Q, Kir J, Liu D, Bryant D, Guo Y, Stephens R, Baseler MW, Lane HC: DAVID Bioinformatics Resources: expanded annotation database and novel algorithms to better extract biology from large gene lists. Nucleic acids research 2007, 35(suppl_2):W169W175. 
40. Smoot ME, Ono K, Ruscheinski J, Wang P-L, Ideker T: Cytoscape 2.8: new features for data integration and network visualization. Bioinformatics 2011, 27(3):431-432.

41. Jiang J, Cole JB, Freebern E, Da Y, VanRaden PM, Ma L: Functional annotation and Bayesian fine-mapping reveals candidate genes for important agronomic traits in Holstein bulls. Communications Biology 2019, 2(1):212.

42. Mahmoudi A, Zargaran A, Amini H-R, Assadi A, Vajdi Hokmabad R, Eghbalsaied S: A SNP in the $3^{\prime}$-untranslated region of AMPKY1 may associate with serum ketone body and milk production of Holstein dairy cows. Gene 2015, 574(1):48-52.

43. Safa A, Bahroudi Z, Shoorei H, Majidpoor J, Abak A, Taheri M, Ghafouri-Fard S: miR-1: A comprehensive review of its role in normal development and diverse disorders. Biomed Pharmacother 2020, 132:110903.

44. Lin H, Chaudhury M, Sharma N, Bhattacharyya S, Elolimy AA, Yeruva L, Ronis MJJ, Mercer KE: MicroRNA profiles were altered in neonatal piglet mammary glands following postnatal infant formula feeding. J Nutr Biochem 2020, 83:108397.

45. Menzies KK, Lefèvre C, Macmillan KL, Nicholas KR: Insulin regulates milk protein synthesis at multiple levels in the bovine mammary gland. Funct Integr Genomics 2009, 9(2):197-217.

46. Brearley MC, Li C, Daniel Z, Loughna PT, Parr T, Brameld JM: Changes in expression of serine biosynthesis and integrated stress response genes during myogenic differentiation of $\mathrm{C2C12}$ cells. Biochem Biophys Rep 2019, 20:100694.

47. Xuan R, Chao T, Wang A, Zhang F, Sun P, Liu S, Guo M, Wang G, Ji Z, Wang J et al: Characterization of microRNA profiles in the mammary gland tissue of dairy goats at the late lactation, dry period and late gestation stages. PLoS One 2020, 15(6):e0234427.

48. Mathews DH, Disney MD, Childs JL, Schroeder SJ, Zuker M, Turner DH: Incorporating chemical modification constraints into a dynamic programming algorithm for prediction of RNA secondary structure. P Natl Acad Sci USA 2004, 101(19):7287-7292.

49. Taganov KD, Boldin MP, Chang KJ, Baltimore D: NF-kappaB-dependent induction of microRNA miR-146, an inhibitor targeted to signaling proteins of innate immune responses. Proc Natl Acad Sci U S A 2006, 103(33):12481-12486.

50. Xiang M, Birkbak NJ, Vafaizadeh V, Walker SR, Yeh JE, Liu S, Kroll Y, Boldin M, Taganov K, Groner $B$ et al: STAT3 induction of miR-146b forms a feedback loop to inhibit the NF-kappaB to IL-6 signaling axis and STAT3-driven cancer phenotypes. Sci Signal 2014, 7(310):ra11.

51. Wicik Z, Gajewska M, Majewska A, Walkiewicz D, Osinska E, Motyl T: Characterization of microRNA profile in mammary tissue of dairy and beef breed heifers. J Anim Breed Genet 2016, 133(1):31-42.

52. Kulig H, Kowalewska-Łuczak I, Żukowski K, Kruszyński W: FABP3, FABP4 and ANXA9 SNP genotypes in relation to breeding values for milk production traits in Polish Holstein-Friesian cows. Russian Journal of Genetics 2013, 49(8):852-856.

53. Sorensen IF, Edwards SM, Rohde PD, Sorensen P: Multiple Trait Covariance Association Test Identifies Gene Ontology Categories Associated with Chill Coma Recovery Time in Drosophila melanogaster. Sci Rep-Uk 2017, 7.

54. Fang LZ, Sorensen P, Sahana G, Panitz F, Su GS, Zhang SL, Yu Y, Li BJ, Ma L, Liu G et al: MicroRNAguided prioritization of genome-wide association signals reveals the importance of microRNA-target gene networks for complex traits in cattle. Sci Rep-Uk 2018, 8.

55. Tiezzi F, Maltecca C, Cecchinato A, Penasa M, Bittante G: Genetic parameters for fertility of 
dairy heifers and cows at different parities and relationships with production traits in first lactation. J Dairy Sci 2012, 95(12):7355-7362.

56. Strucken EM, Bortfeldt RH, Tetens J, Thaller G, Brockmann GA: Genetic effects and correlations between production and fertility traits and their dependency on the lactation-stage in Holstein Friesians. BMC Genet 2012, 13:108-108. 
603 Table 1. The differentially expressed miRNAs between high and low milk protein 604 content in lactation and non-lactation.

\begin{tabular}{|c|c|c|}
\hline Group & $\begin{array}{l}\text { DE miRNAs } \\
\text { number }\end{array}$ & DE miRNA list \\
\hline HP vs LP & 28 & $\begin{array}{l}\text { let-7a-3p, let-7f, miR-143, miR-144, miR-146a, miR-146b, miR-150, miR-152, } \\
\text { miR-16a, miR-185, miR-204, miR-24-3p, miR-2478, miR-2904, miR-320a, } \\
\text { miR-326, miR-340, miR-34a, miR-374a, miR-379, miR-382, miR-411c-3p, } \\
\text { miR-423-3p, miR-423-5p, miR-425-3p, miR-451, miR-655, miR-92b }\end{array}$ \\
\hline HD vs LD & 52 & $\begin{array}{l}\text { miR-1, miR-100, miR-10a, miR-10b, miR-1185, miR-1271, miR-1296, miR- } \\
\text { 132, miR-1388-5p, miR-141, miR-143, miR-144, miR-146a, miR-146b, miR- } \\
\text { 147, miR-149-5p, miR-150, miR-152, miR-16a, miR-185, miR-192, miR-195, } \\
\text { miR-196a, miR-196b, miR-199a-5p, miR-20b, miR-24-3p, miR-2411-3p, miR- } \\
\text { 2478, miR-26a, miR-26b, miR-27b, miR-2887, miR-2904, miR-296-3p, miR- } \\
\text { 30a-5p, miR-326, miR-331-3p, miR-374a, miR-379, miR-409a, miR-429, miR- } \\
\text { 484, miR-486, miR-494, miR-495, miR-505, miR-6524, miR-665, miR-885, } \\
\text { miR-99a-5p, miR-99b }\end{array}$ \\
\hline Common & 14 & $\begin{array}{l}\text { miR-143, miR-144, miR-146a, miR-146b, miR-150, miR-152, miR-16a, miR- } \\
\text { 185, miR-24-3p, miR-2478, miR-2904, miR-326, miR-374a, miR-379 }\end{array}$ \\
\hline
\end{tabular}

605

606 Table 3. The differentially expressed miRNAs with their potential target genes related

607 to milk protein synthesis

\begin{tabular}{|l|l|l|}
\hline \multicolumn{1}{|c|}{ miRNAs } & \multicolumn{1}{c|}{ Targets } & Group \\
\hline let-7a-3p & BAMBI, COL4A5, DNAJC6 & HP vs LP \\
\hline miR-144, miR-150, miR-16a, miR-2478 & MYB & HP vs LP \\
\hline miR-146a, miR-146b & ALOX15, FABP3 & HP vs LP \\
\hline miR-2478 & ME3, MYB & HP vs LP \\
\hline miR-2904 & DNAJC6 & HP vs LP \\
\hline miR-374a & MYB, DUSP13 & HP vs LP \\
\hline miR-1 & PSPH & HD vs LD \\
\hline miR-1296 & CCNB2 & HD vs LD \\
\hline miR-141 & SMAD9, ITGA8, ATP6V0D2, MAD2L1 & HD vs LD \\
\hline miR-195, miR-16a & CCNB2 & HD vs LD \\
\hline miR-1271, miR-196a, miR-196b, miR-152 & COL2A1 & HD vs LD \\
\hline miR-2887 & ANGPT4 & HD vs LD \\
\hline miR-429 & SPP1 & HD vs LD \\
\hline miR-505 & ACSBG1 & HD vs LD \\
\hline
\end{tabular}




\begin{tabular}{|l|l|l|}
\hline miR-885 & NR1D1 & HD vs LD \\
\hline miR-144 & ASF1B, SPP1, CDK1 & HD vs LD \\
\hline miR-146a, miR-146b & FABP3 & HD vs LD \\
\hline miR-185 & SPP1, FABP3 & HD vs LD \\
\hline miR-2478 & KCNJ2 & HD vs LD \\
\hline miR-2904 & PODN, SFRP1 & HD vs LD \\
\hline
\end{tabular}

608

$609 \quad$ Figure 


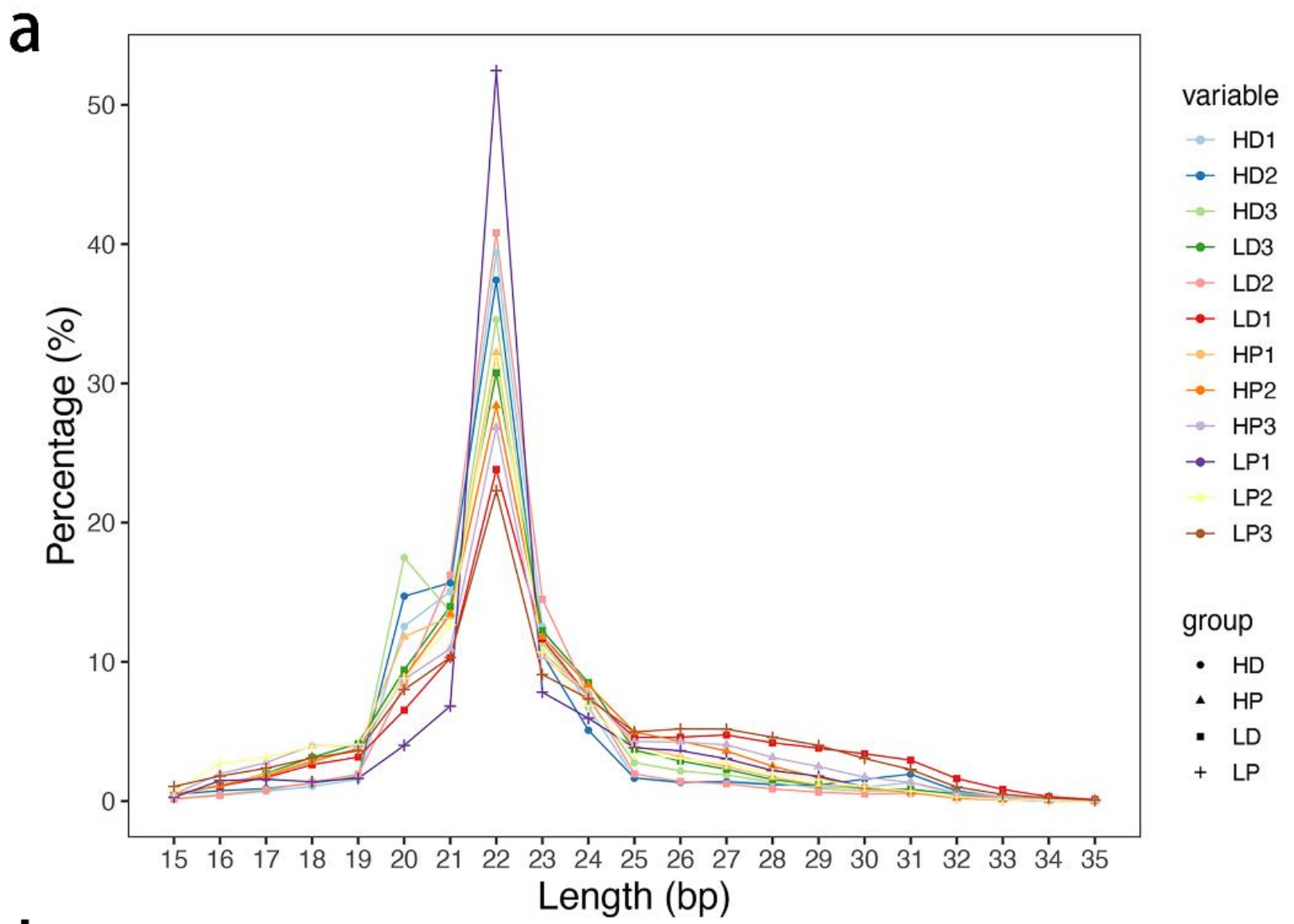

b

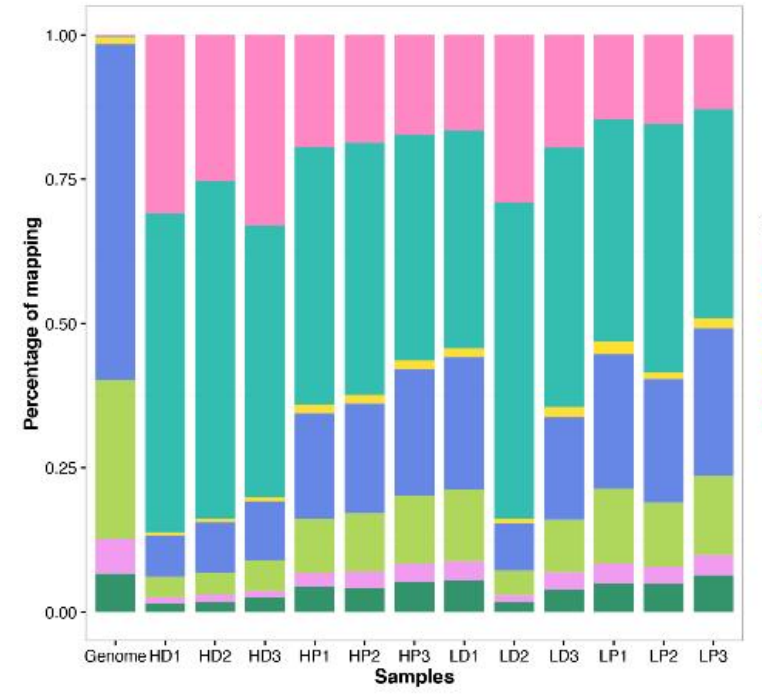

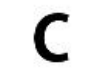

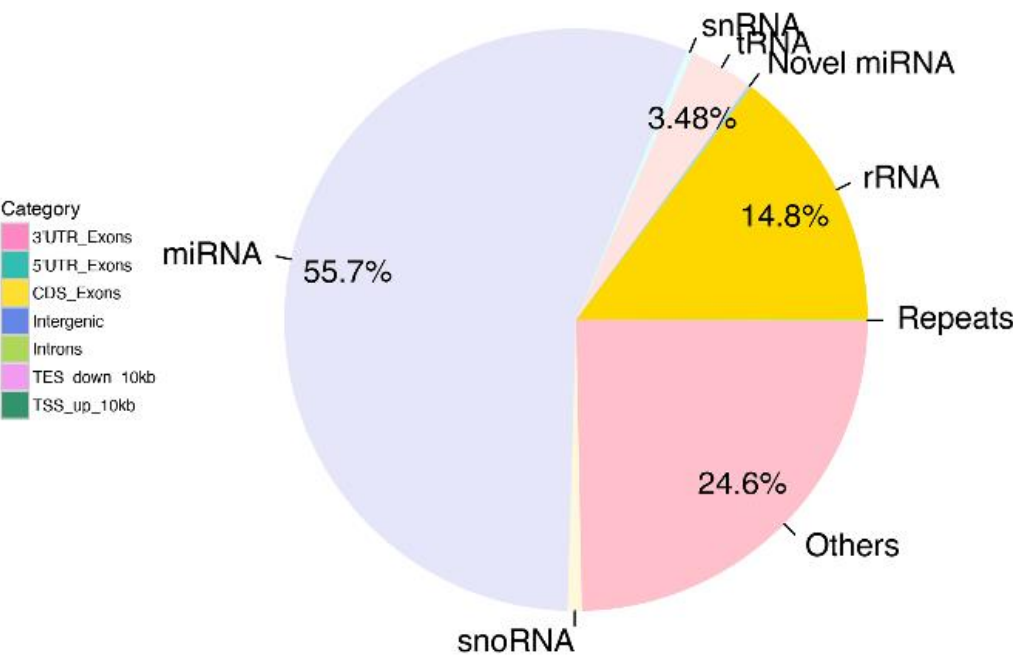

, sn $48 \%$

$14.8 \%$ Repeats

611 Figure 1. Mapping statistics. (a) Length distribution of the mapped reads across all the

612 libraries. (b) The genome distributions of the mapped reads for all samples. (c) The

613 relative abundance of different classes of small RNAs in the total reads was successfully mapped to the bovine genome. 
a
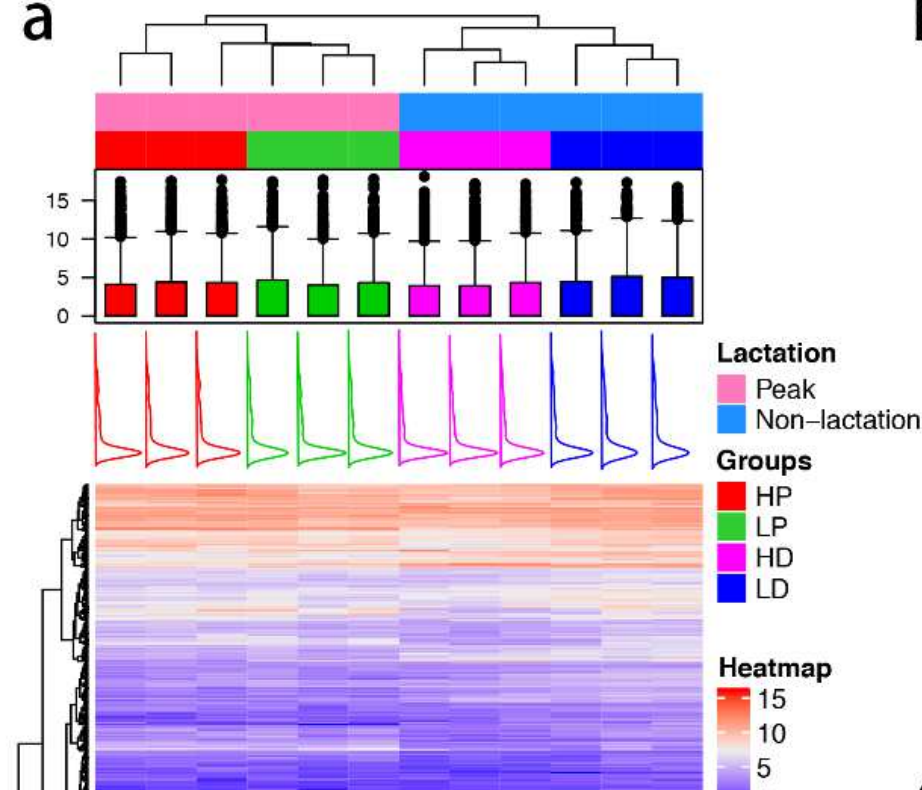

C

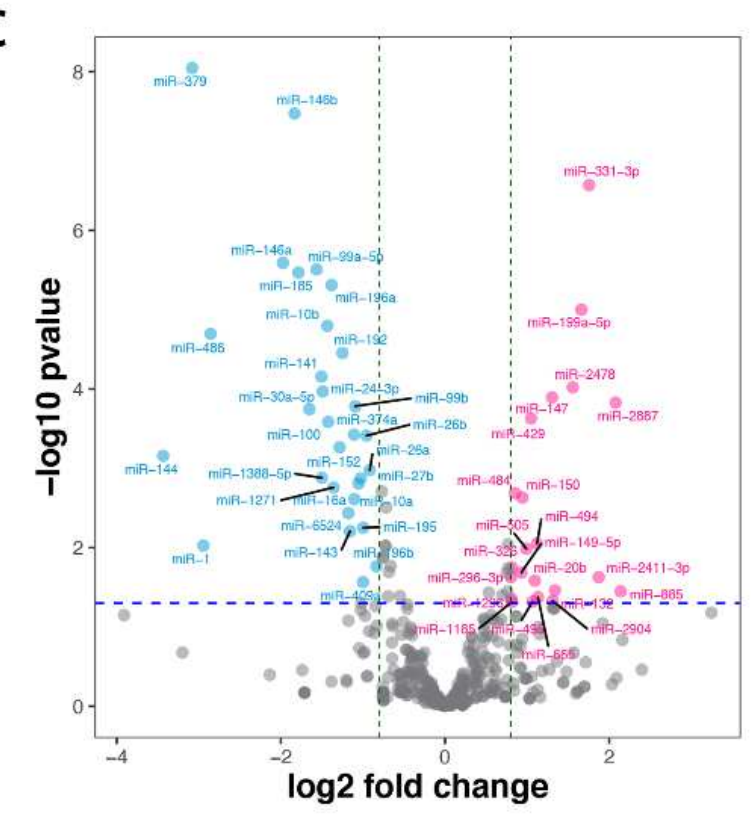

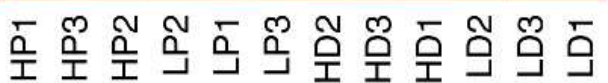

color_flag

- Down

- Not significant

- Up color_flag

- Down

- Not significant

- Up

615

616 Figure 2. The expression heatmap and volcano plot of DE miRNAs. (a) Cluster analysis

617 of all miRNAs based on their expression. Red indicated higher expression, and blue

618 showed lower expression. (b) Volcano plot displaying differentially expressed miRNAs

619 of HP vs. LP. The red and blue dots represent the significantly upregulated and

620 downregulated miRNA; the gray dots represent miRNAs whose expression levels do

621 not reach statistical significance. (c) Volcano plot displaying differentially expressed

622 miRNAs of HP vs. LP. 

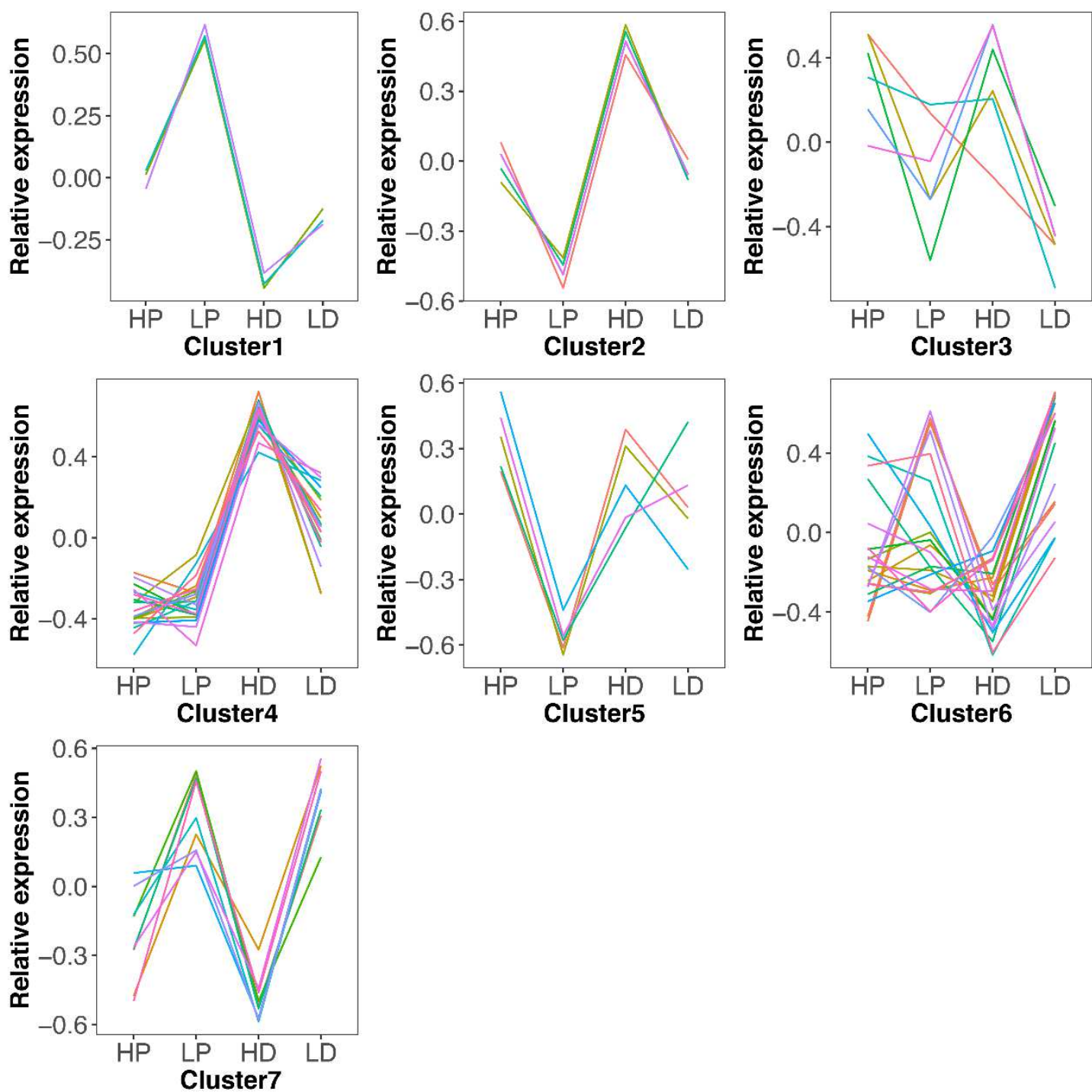

623

Cluster7

624 Figure 3. The expression pattern of common DE miRNAs using k-means clustering 625 with Pearson correlation. 

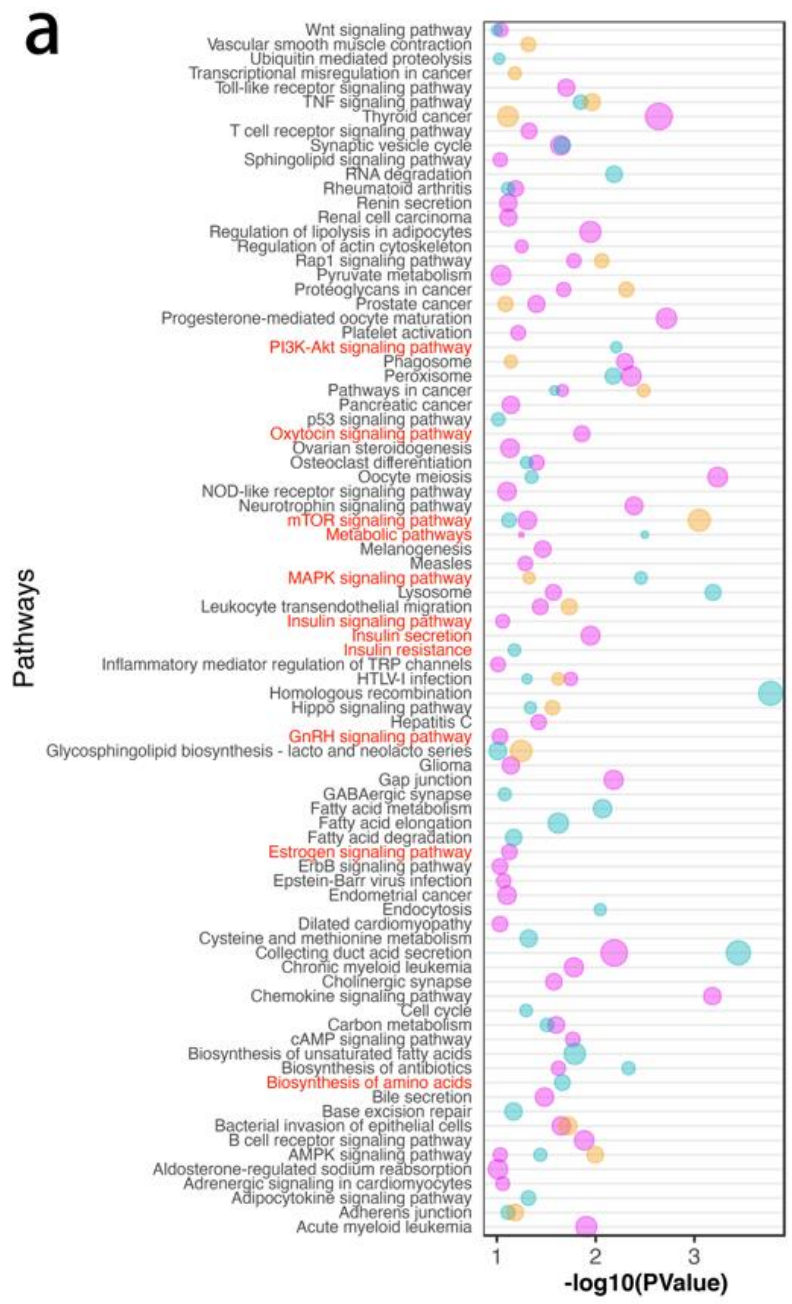

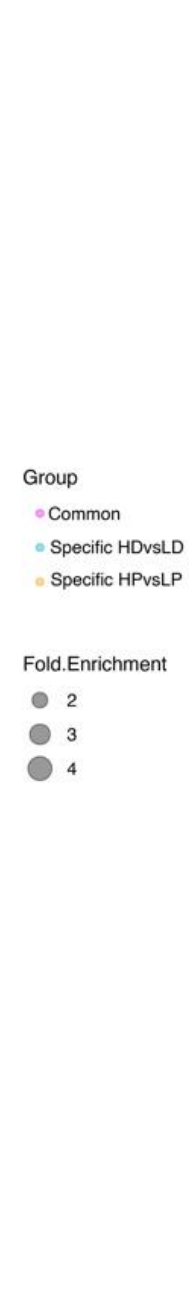

b

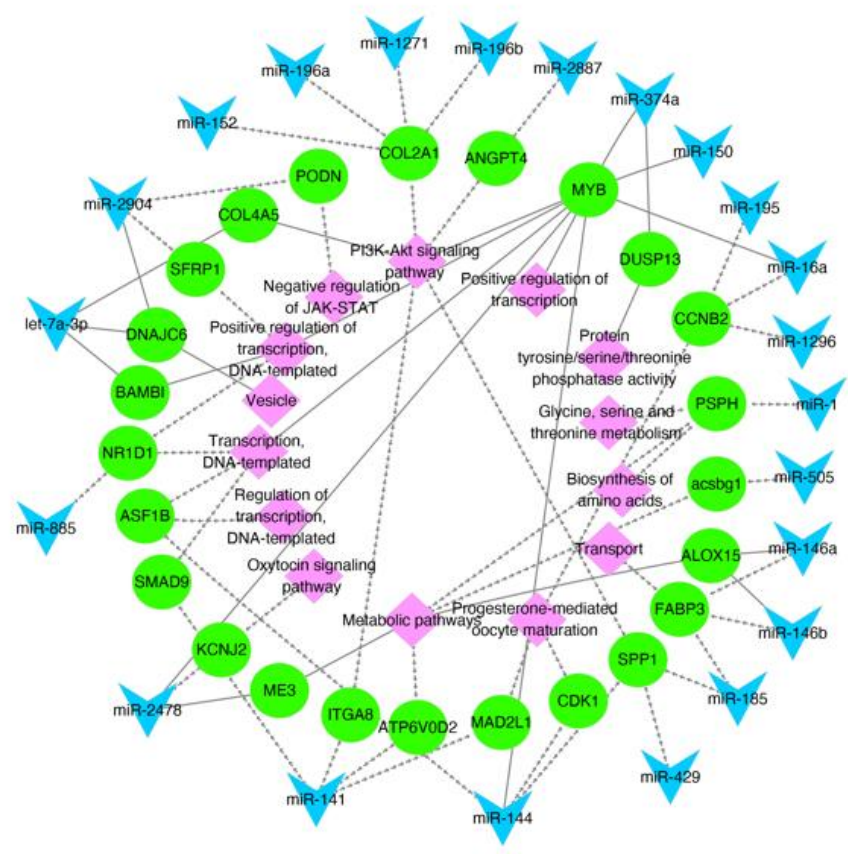

626

627 Figure 4. The functional annotation of using k-means clustering with Pearson

628 correlation. (a) Predominant function categories targeted by common DE miRNAs,

629 lactation-specific DE miRNAs, and non-lactating-specific DE miRNAs. More

630 significant values and shapes were suggesting higher relevance and higher enriched

631 fold, respectively. (b) Network plot of candidate miRNAs, mRNAs, and pathways. The

632 blue triangles, green circles, and pink diamonds represent miRNAs, mRNAs, and

633 pathways, respectively. The dashed and solid lines represent the lactating and non-

634 lactating period networks, respectively. 

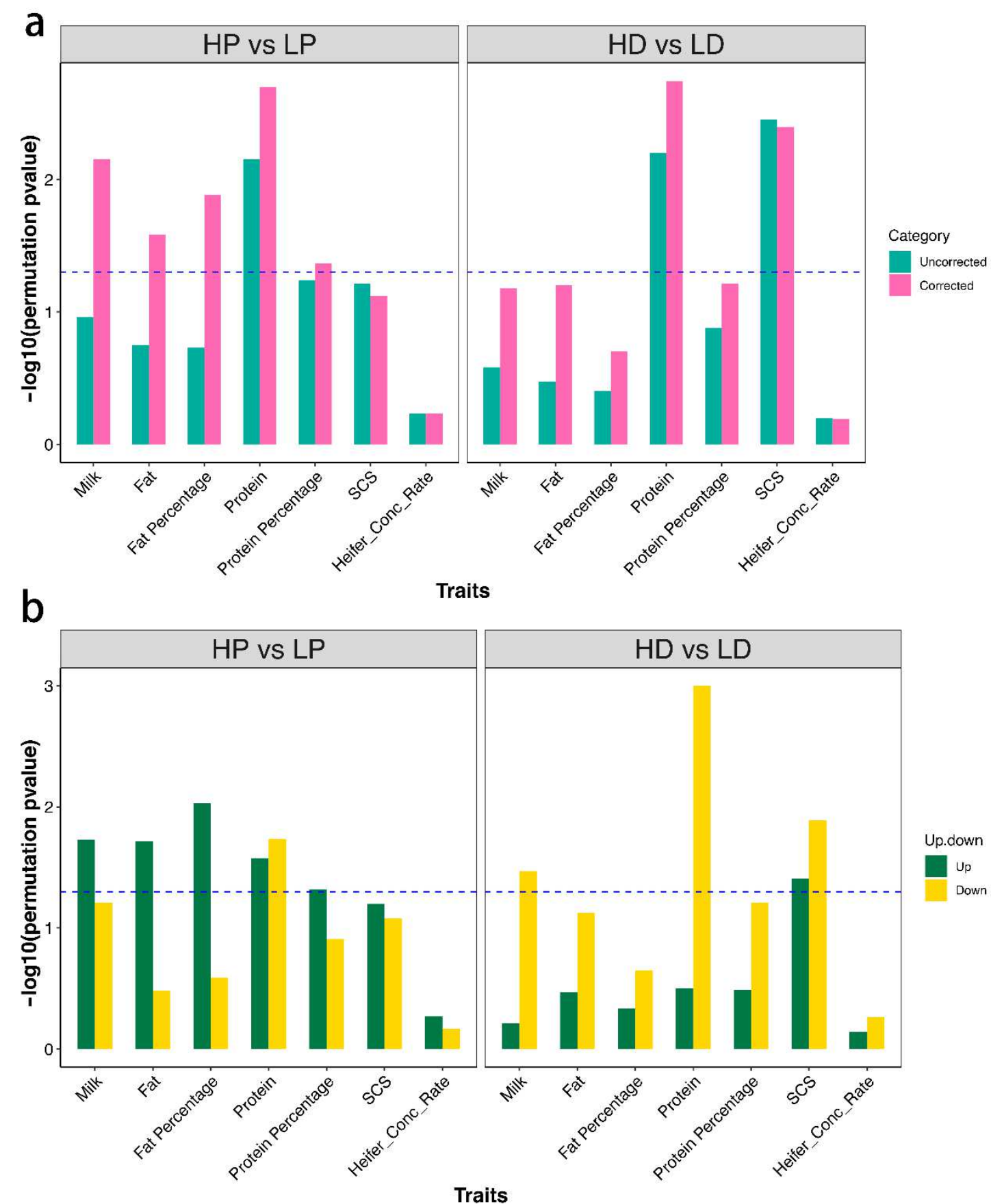

Figure 5. The GWAS enrichment for targets of DE miRNAs in different groups. (a)

The enrichment for targets of DE miRNAs in HP vs. LP and HD vs. LD. The green bars represent the p-values of permutation tests using original GWAS statistics. The red bars represent the p-values of permutation tests using corrected GWAS statistics (remove SNPs around the DGATl gene). (b) The enrichment for targets of upregulated and 641 downregulated DE miRNAs in HP vs. LP and HD vs. LD. The dark green and gold color represent the p-values of permutation tests for the upregulated and downregulated DE miRNAs, respectively. 


\section{Supplementary information}

645 Tables

646 Table S1: The information of small RNA sequencing for 12 Chinese Holstein cattle.

647 Table S2: Summary of sequence reads alignment.

648 Table S3: The locations and expressions of the identified novel miRNAs.

649 Table S4: The information of differentially expressed miRNAs in HP vs. LP and HD 650 vs. LD.

651 Table S5: The functional annotation of targets of DE miRNAs

652

$653 \quad$ Figures

654 Figure S1: The expression of the top 20 associate miRNAs in lactation and non655 lactation period.

656 


\section{Figures}

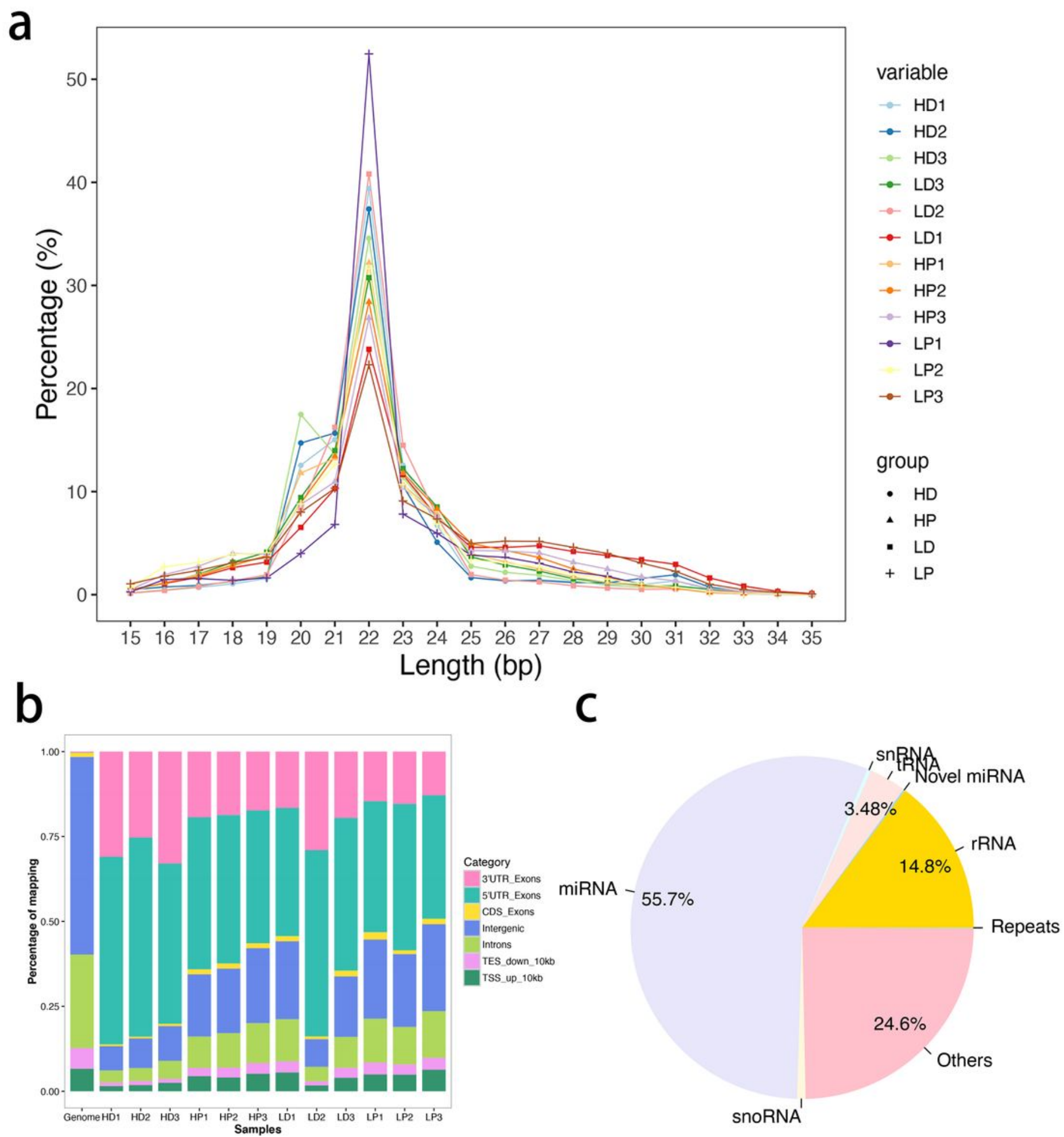

\section{Figure 1}

Mapping statistics. (a) Length distribution of the mapped reads across all the libraries. (b) The genome distributions of the mapped reads for all samples. (c) The relative abundance of different classes of small RNAs in the total reads was successfully mapped to the bovine genome. 
a
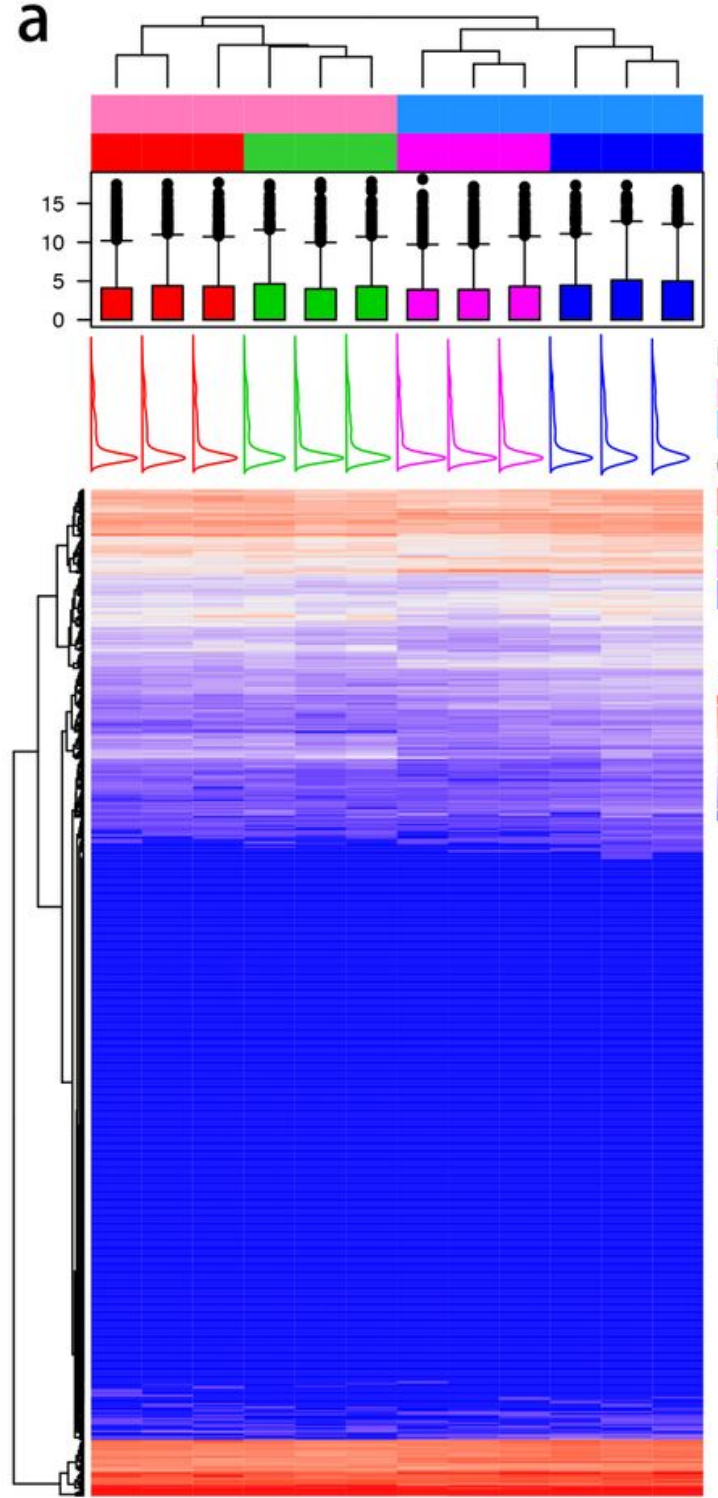

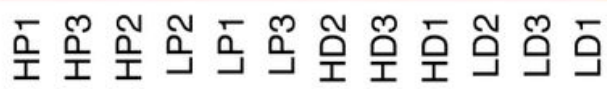

b

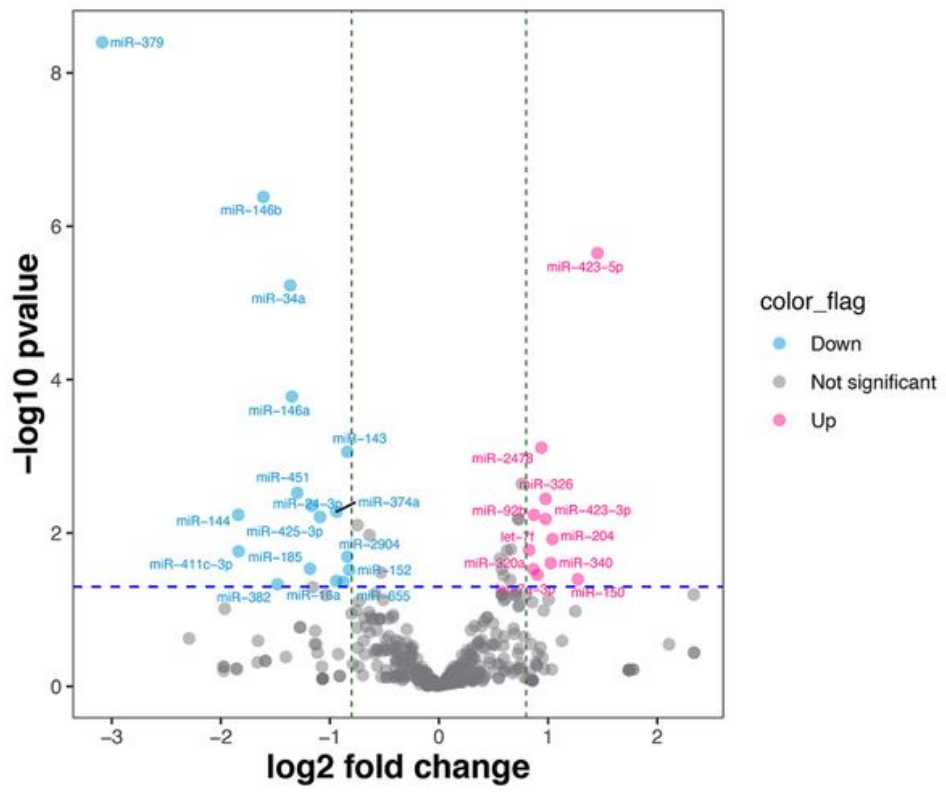

C

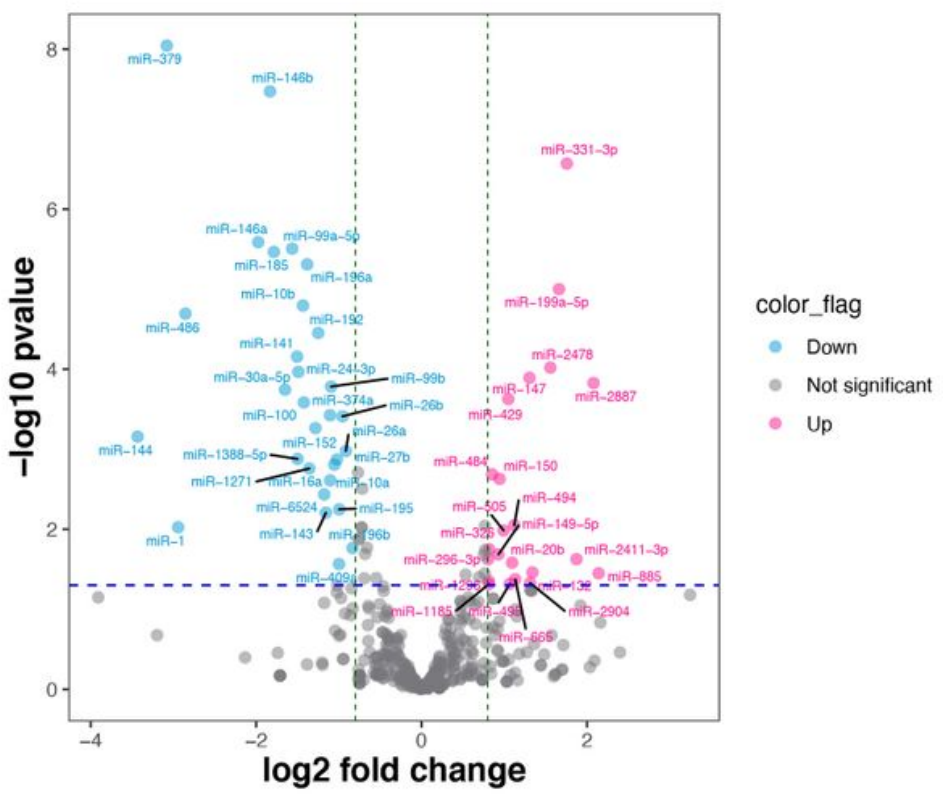

Figure 2

The expression heatmap and volcano plot of DE miRNAs. (a) Cluster analysis of all miRNAs based on their expression. Red indicated higher expression, and blue showed lower expression. (b) Volcano plot displaying differentially expressed miRNAs of HP vs. LP. The red and blue dots represent the significantly upregulated and downregulated miRNA; the gray dots represent miRNAs whose expression levels do not reach statistical significance. (c) Volcano plot displaying differentially expressed miRNAs of HP vs. LP. 

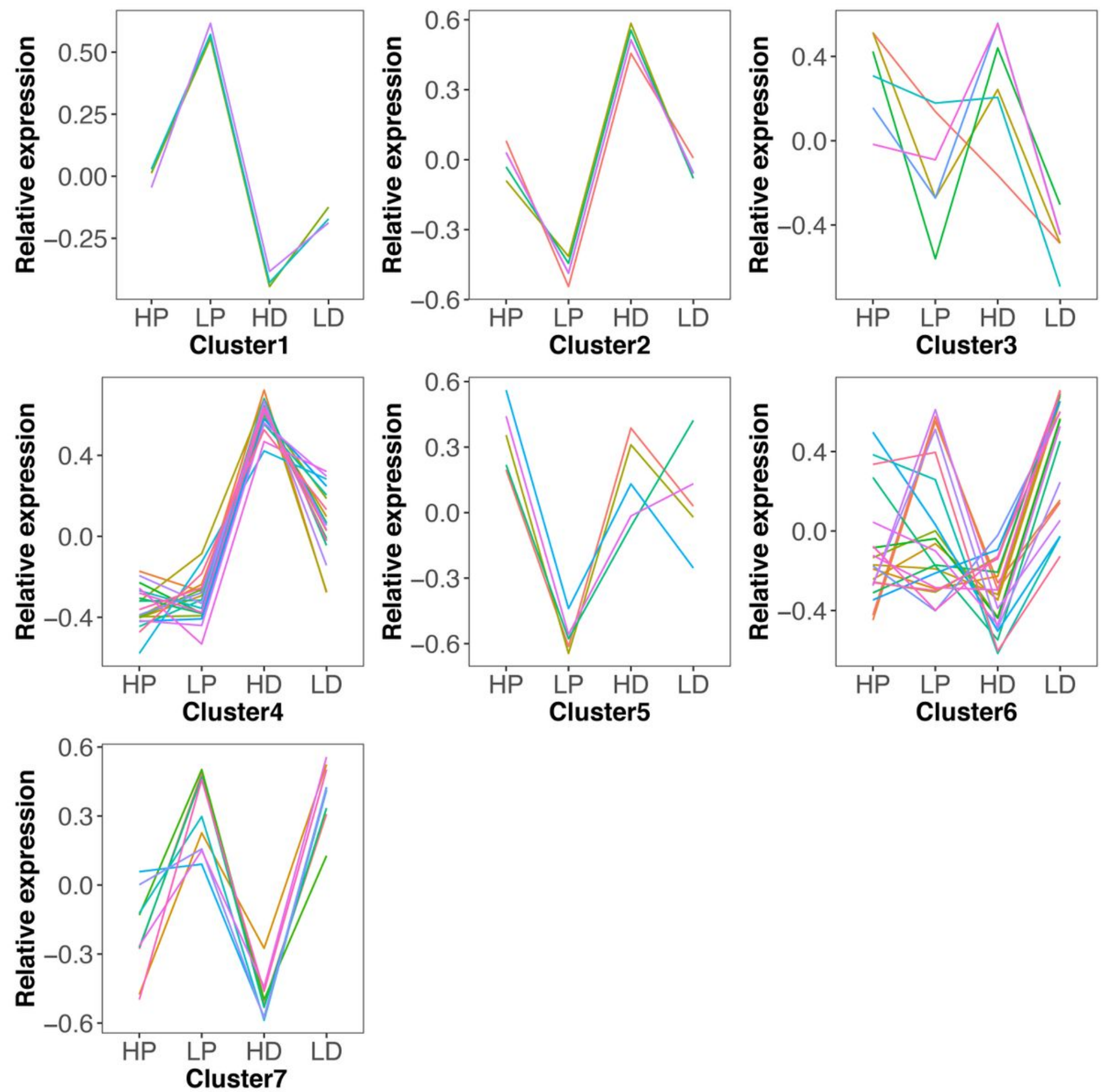

Figure 3

The expression pattern of common DE miRNAs using k-means clustering with Pearson correlation. 


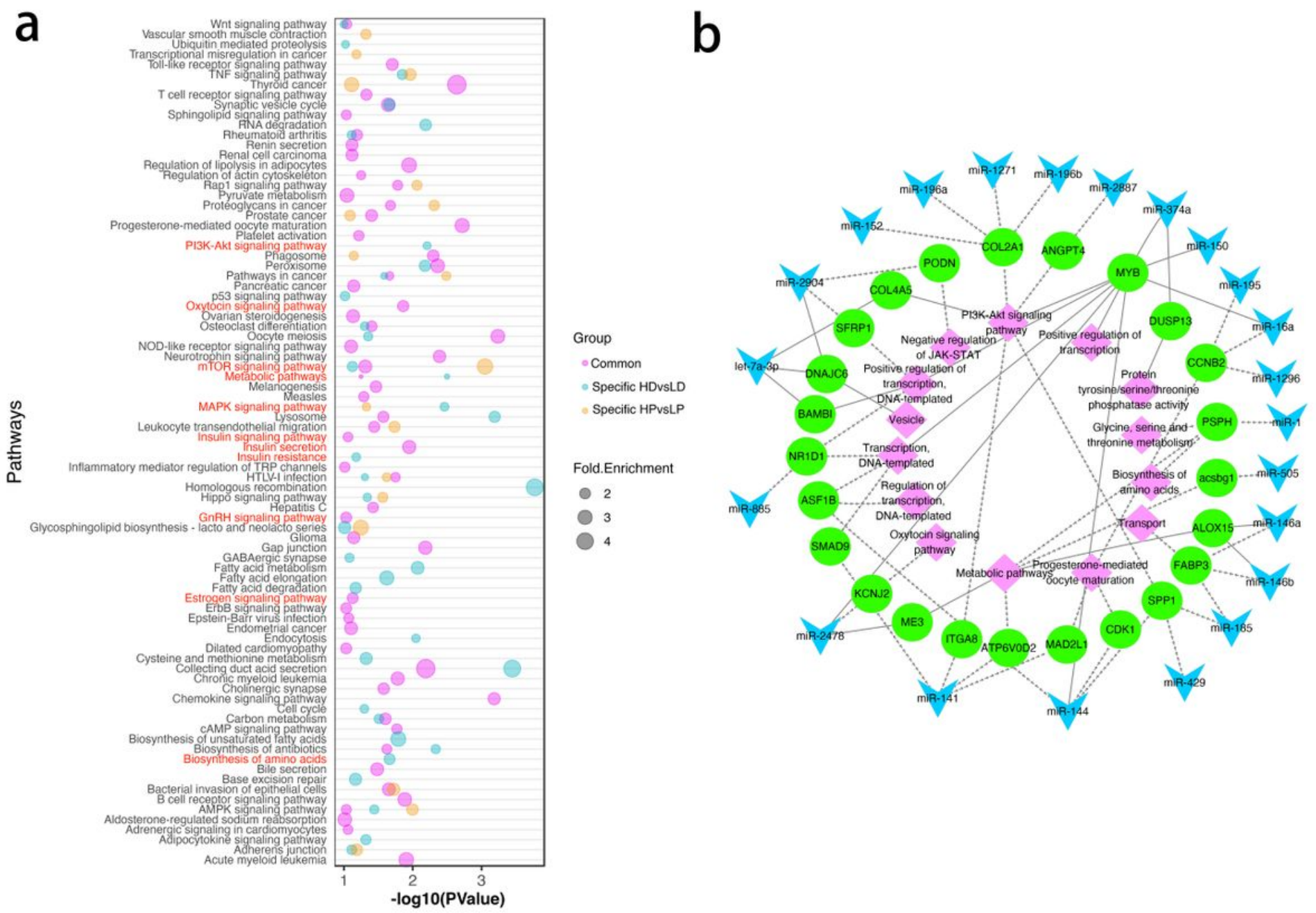

Figure 4

The functional annotation of using k-means clustering with Pearson correlation. (a) Predominant function categories targeted by common DE miRNAs, lactation-specific DE miRNAs, and non-lactatingspecific DE miRNAs. More significant values and shapes were suggesting higher relevance and higher enriched fold, respectively. (b) Network plot of candidate miRNAs, mRNAs, and pathways. The blue triangles, green circles, and pink diamonds represent miRNAs, mRNAs, and pathways, respectively. The dashed and solid lines represent the lactating and non-lactating period networks, respectively. 


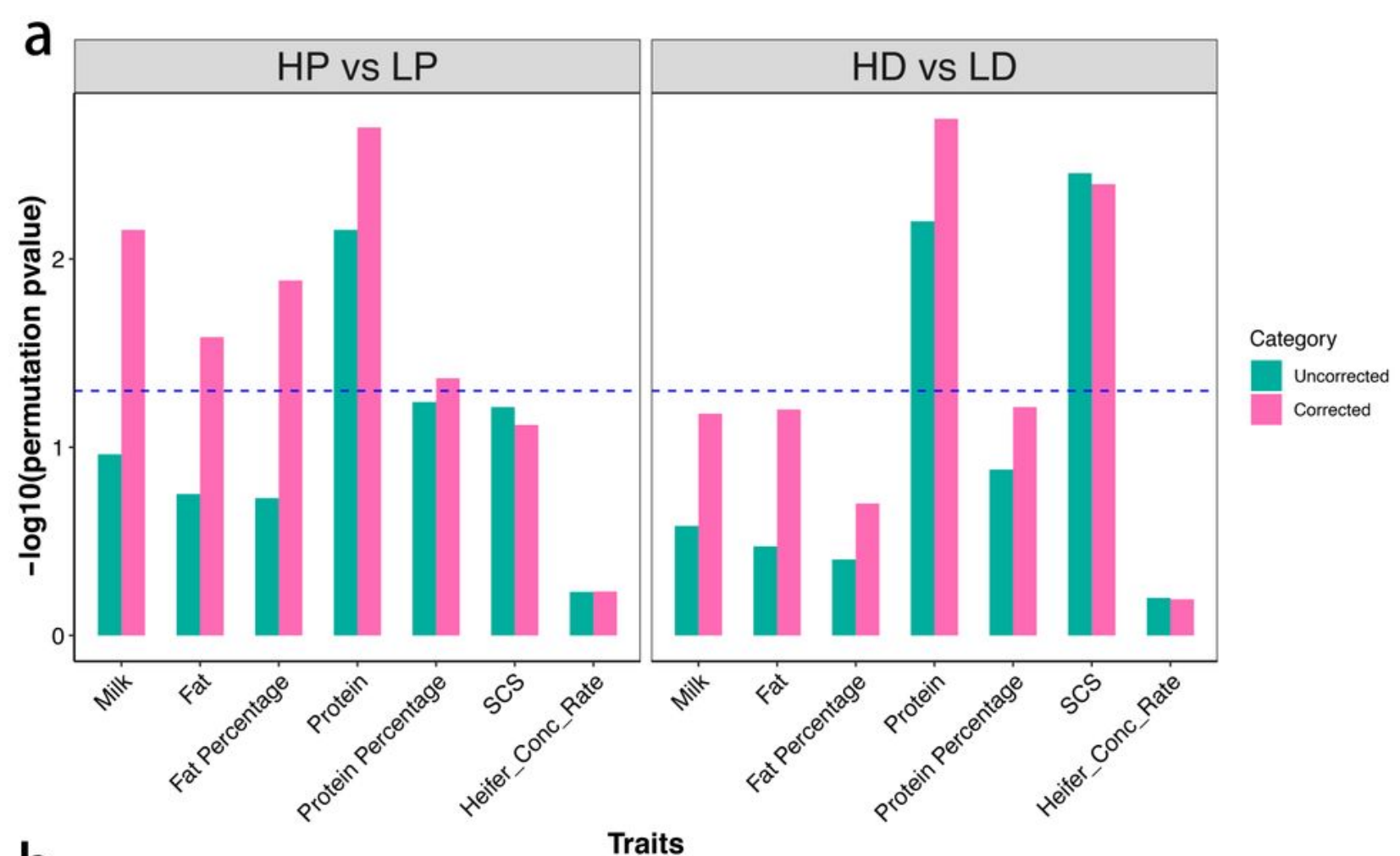

b

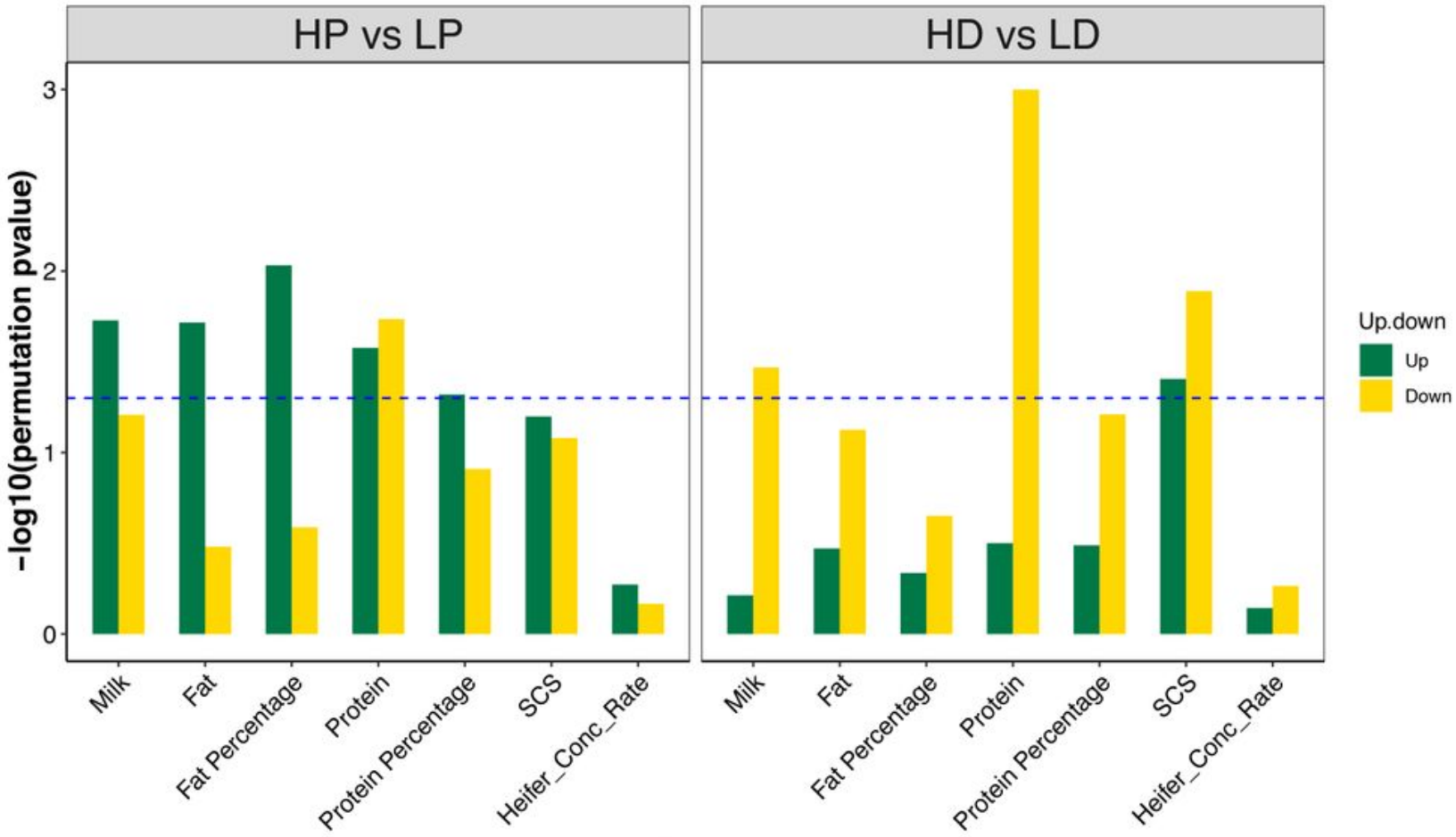

Traits

Figure 5

The GWAS enrichment for targets of DE miRNAs in different groups. (a) The enrichment for targets of DE miRNAs in HP vs. LP and HD vs. LD. The green bars represent the p-values of permutation tests using original GWAS statistics. The red bars represent the p-values of permutation tests using corrected GWAS statistics (remove SNPs around the DGAT1 gene). (b) The enrichment for targets of upregulated and 
downregulated DE miRNAs in HP vs. LP and HD vs. LD. The dark green and gold color represent the $\mathrm{p}-$ values of permutation tests for the upregulated and downregulated DE miRNAs, respectively.

\section{Supplementary Files}

This is a list of supplementary files associated with this preprint. Click to download.

- FigureS1.tif

- TableS1.xlsx

- Tables2.xlsx

- TableS3.xlsx

- TableS4.xlsx

- TableS5.xIsx 